\title{
Running-based pica and taste avoidance in rats
}

\author{
Sadahiko Nakajima ${ }^{1}$
}

Published online: 9 November 2017

(C) Psychonomic Society, Inc. 2017

\begin{abstract}
Running in an activity wheel generates pica behavior (kaolin clay intake) in rats. Wheel running also results in Pavlovian conditioned avoidance of the taste solution consumed immediately before the running. Since pica has been considered a behavioral marker of nausea in rats, these findings suggest that wheel running induces nausea, which is the underlying physiological state for establishing taste avoidance. This article reports a replication of running-based pica in rats (Experiment 1) and concurrent demonstrations of running-based pica and taste avoidance in the same animals (Experiments 2 and 3). Also shown is that pica does not alleviate running-based taste avoidance (Experiment 3). Another finding is that pica is generated by a nausea-inducing lithium chloride injection but not by a pain-inducing hypertonic saline injection (Experiment 4). These results, when taken together, support the hypothesis that pica behavior generated by wheel running reflects nausea in rats.
\end{abstract}

Keywords Pica · Taste aversion - Wheel running - Nausea . Rat

Aberrant pica behavior (kaolin clay intake) has been regarded as a marker of nausea in rats (Andrews \& Horn, 2006) that cannot vomit because of anatomical and/or neural reasons (Horn et al., 2013). Pica behavior is generated by a variety of nausea-inducing treatments, including irradiation (e.g., Yamamoto, Takeda, \& Yamatodani, 2002), motion sickness (e.g., Mitchell, Laycock, \& Stephens, 1977), and

Sadahiko Nakajima

nakajima@kwansei.ac.jp

1 Department of Psychological Science, Kwansei Gakuin University, Nishinomiya 662-8501, Japan administration of emetic drugs such as lithium chloride (LiCl; e.g., Mitchell et al., 1976), cyclophosphamide (e.g., Tohei, Kojima, Ikeda, Hokao, \& Shinoda, 2011), cisplatin (e.g., Takeda, Hasegawa, Morita, \& Matsunaga, 1993), ritonavir (e.g., Aung et al., 2005), morphine (e.g., Aung, Mehendale, Xie, Moss, \& Yuan, 2004), and apomorphine (e.g., Takeda et al., 1995), among others (see the introduction of Nakajima, 2016a, for a more detailed review).

Recent studies conducted in my laboratory have shown that pica is also generated in rats by voluntary and forced running in activity wheels (Nakajima, 2016a; Nakajima \& Katayama, 2014), suggesting that running induces visceral discomfort similar to nausea. This finding is consonant with the fact that, similar to emetic drugs conventionally employed in Pavlovian conditioned taste-aversion studies in rats, ${ }^{1}$ wheel running acts as an agent (i.e., an unconditioned stimulus; US) to establish avoidance of a paired taste (e.g., Heth, Inglis, Russell, \& Pierce, 2001; Lett \& Grant, 1996; Nakajima, Hayashi, \& Kato, 2000; see Boakes \& Nakajima, 2009, for a review). Notably, running-based taste avoidance is prevented by the administration of anti-emetic granisetron (Eccles, Kim, \& O'Hare, 2005), supporting the hypothesis that nausea is the underlying physiological state for establishing running-based taste avoidance. In addition, the finding that running-based taste avoidance is alleviated not only by previous experience of running but also by prior injection of emetic $\mathrm{LiCl}$ (i.e., cross-US familiarization effect: Nakajima, Urata, \& Ogawa, 2006) implies that a common process (presumably nausea) is

\footnotetext{
${ }^{1}$ In the present article, the term taste avoidance is used for suppression of a target taste intake, while the term taste aversion is reserved for the case when its underlying physiological state is identified as nausea (Parker, 2003, 2014). Although some researchers (e.g., Lin, Arthurs, \& Reilly, 2014, 2017) have limited taste avoidance to intake suppression not based on nausea, this term is used here in a broader sense, regardless of the underlying physiological states.
} 
physiologically habituated by preexposure. Furthermore, a unique reduction in taste palatability, measured by licking patterns (Baird, St John, \& Nguyen, 2005; Dwyer, 2009, 2012), accompanies both running-based and LiCl-based taste avoidances, implying that running and $\mathrm{LiCl}$ commonly induce nausea (Dwyer, Boakes, \& Hayward, 2008; but see Lin, Arthurs, Amodeo, \& Reilly, 2012, for a dissenting view). Finally, aversive orofacial responses, which also reflect taste palatability (Grill \& Norgren, 1978), are invoked by the taste paired with wheel running, at least when the running is accompanied with a rocking movement of the wheel (Grant et al., 2012). This suggests motion sickness may be produced by wheel running, because motion sickness produces aversive taste reactivity responses as well (Ossenkopp et al., 2003).

The present research, consisting of four experiments, attempts to further clarify the nature of running-based pica and taste avoidance in rats. The general methods of the present research, including rats' strain and age, experimental setup, and principal data analysis, were the same as in the previous studies (Nakajima, 2016a; Nakajima \& Katayama, 2014). Experiment 1 was conducted to replicate pica based on voluntary wheel running in order to determine the proper number of running days for the following experiments. Experiment 2 was designed for a concurrent demonstration of runningbased pica and taste avoidance. The reasoning discussed in the preceding paragraph leads us to expect that wheel running produces pica and taste avoidance (intake suppression) in the same animals, because it is assumed that nausea is a common cause of these behaviors. Experiment 2 also explored correlations between the amounts of pica and taste avoidance.

Experiment 3 was planned to explore the functional role of pica in running rats. One may consider that pica is an adaptive response to dietary toxin (De Jonghe, Lawler, Horn, \& Tordoff, 2009), because kaolin clay absorbs toxin (Dominy, Davoust, \& Minekus, 2004) and prevents diarrhea (Beck, Jenkins, Thurber, \& Ambrus, 1977). If such a remedial effect of pica is applicable to discomfort induced by running, then kaolin intake may alleviate running-based taste avoidance because it is expected that the attenuated discomfort has a weakened potential to establish taste avoidance. Experiment 3 tested this possibility by measuring running-based taste avoidance in rats that could consume kaolin and rats without kaolin. If kaolin has some alleviating (or remedial) effect on runninginduced discomfort, then the former rats should show weaker taste avoidance than the latter rats.

The aim of Experiment 4 was to assess the validity of pica as a behavioral marker of nausea. In rats, conditioned taste avoidance can be established not only by emetic agents but also by nonemetic stressful events such as dermal pain by electric shocks (e.g., Garcia, Kovner, \& Green, 1970; Krane \& Wagner, 1975; Nakajima, 2004) and visceral pain by intraperitoneal (ip) injections of hypertonic saline (e.g., Hargrave \& Bolles, 1971; Lin, Arthurs, \& Reilly, 2013; Sakai \&
Yamamoto, 1997). Thus, demonstration of taste avoidance does not, in itself, necessarily guarantee that rats experience nausea (Grant, 1987; Parker, 2003, 2014; Parker, Limebeer, \& Rana, 2009). In the same vein, any stressful event or any visceral discomfort might generate pica in rats. Experiment 4 tested this possibility by monitoring kaolin intake during the conditioning phase where ip injections of pain-inducing hypertonic saline followed a target taste solution. If rats show no pica behavior, though displaying taste avoidance, then it validates pica as a marker of nausea, because the hypertonic saline would induce little nausea in rats. Furthermore, it indirectly supports the claim that wheel running induces nausea in rats, because running generates not only taste avoidance but also pica.

\section{Experiment 1}

The primary aim of Experiment 1 was to determine the proper number of daily sessions to obtain reliable pica with 45-min wheel running for the subsequent experiments. A subsidiary aim of this experiment was to address the role of contextual cues in carryover effects of running-based pica. Rats consumes some, though small, kaolin, even after wheel running becomes unavailable (Nakajima \& Katayama, 2014). A possible account of this carryover effect is Pavlovian conditioning of running-induced nausea to the environmental cues: Rats may feel malaise when reencountering such cues, resulting in kaolin intake (cf. Mitchell, Winter, \& Morisaki, 1977). Although this account has already been rejected in Nakajima (2016a) by reporting carryover effect without any reliable environmental cues for wheel running, it was tested here again, but with a different test procedure: the rats were exposed to either locked wheels or neutral cages on the postrunning days. Equivalent small kaolin intakes would provide another piece of evidence that the contextual cues plays little or no role in the carryover effect. Furthermore, it would collaborate the premise that running, rather than simple confinement in the wheels, is critical to generate pica.

\section{Method}

\section{Subjects and apparatus}

Eight experimentally naïve male rats (Slc: Wistar/ST) purchased from a supplier (Japan SLC, Inc., Shizuoka, Japan) were housed in individual wire home cages $(20 \mathrm{~cm}$ wide, $25 \mathrm{~cm}$ long, and $18.7 \mathrm{~cm}$ high) in a vivarium on a $16: 8$-hour light-dark cycle (lights on at 0800 ) at $23^{\circ} \mathrm{C}$ and $55 \%$ humidity. They were 9 weeks old on the first day of the experiment. Throughout the experiment, water was freely available from a built-in stainless nozzle protruding through a hole in the center of the back wall of each cage. 
Each animal was supplied with cylindrical food pellets (MF diet, Oriental Yeast Co. Ltd., Tokyo, Japan) available ad libitum from a stainless container $(7.5 \mathrm{~cm}$ wide, $4.5 \mathrm{~cm}$ long, and $15 \mathrm{~cm}$ deep) positioned inward with its end apertures $3.5 \mathrm{~cm}$ above the cage floor. Kaolin pellets were available ad libitum for all rats. The kaolin pellets were composed of kaolin powder and gum arabic at a 99:1 (w/w) ratio; they were mixed with tap water to form cylindrical pellets and completely dried at room temperature of $23^{\circ} \mathrm{C}$. A hanging stainless container ( $8 \mathrm{~cm}$ wide, $4.5 \mathrm{~cm}$ long, and $6 \mathrm{~cm}$ deep) filled with kaolin pellets was installed next to the food container, with its end apertures $10 \mathrm{~cm}$ above the cage floor. A plastic tray ( $22.5 \mathrm{~cm}$ wide, $32 \mathrm{~cm}$ long, and $5.5 \mathrm{~cm}$ deep) with paper bedding was positioned $10 \mathrm{~cm}$ below each cage to collect excreta, food shatters, and kaolin splinters.

After daily measurement of body weights, the rats were transferred, by a carrying cart having individual compartments, to a conventionally illuminated experimental room nearby, which had eight handmade metal activity wheels (15 cm wide and $30 \mathrm{~cm}$ diameter) hung on a wall in two rows and four columns. The sides of the wheel were perforated metal sheets, and the running surface was made of $0.2-\mathrm{cm}$ metal rods spaced $1 \mathrm{~cm}$ apart. The wheels could be tuned in both directions. The minimum torque to initiate the movement when the forepaws of animal were $10 \mathrm{~cm}$ from the lowest point of the wheel was $25 \mathrm{cN}$ measured by a Correx tension gauge (Haag-Streit A.G., Koeniz, Switzerland). A full turn of each wheel was counted automatically by a handcrafted system consisting of a small magnet on the outer rim of the wheel, a reed switch, and an electric pedometer. Each wheel could be locked by two plastic tied laundry pinches.

\section{Procedure}

The protocols of this and following experiments were administered by laboratory assistants who were unaware of the purpose of the study. In this experiment, all experimental procedures were administered with a single squad of eight rats on successive days.

At 1030 of each day of this experiment, all rats were weighed and then moved to the individual compartments of the cart. The cart was kept in the vivarium for $45 \mathrm{~min}$ on the initial 4 baseline days (Days 1-4). On the next 8 days (Days $5-12$ ), animals in the cart were transferred to the experimental room, where all rats were allowed to run in the unlocked wheels for $45 \mathrm{~min}$. The travelling time from the vivarium to the experimental room was around $1 \mathrm{~min}$. The rats were then returned to the no-running condition for the next 4 days (Days 13-16), but this time they were confined into either the locked wheels or the associatively neutral, empty cages (i.e., copies of the home cages without food nor kaolin) during the 45-min period. The order of confinement was counterbalanced: half of the rats were kept in the locked wheels on the odd days (Days
13 and 15) and in the empty cages on the even days (Days 14 and 16), while the order was reversed for the other half.

\section{Measurement and analysis}

The amounts of food and kaolin consumed in the home cages (i.e., 23-hour intakes) were recorded every day by removing the food and kaolin containers, immediately after the rats were moved to the experimental room. The containers were weighed with an electric balance to the nearest $0.1 \mathrm{~g}$, refilled, and replaced before the rats were returned to the home cages. Crushed kaolin and food in the tray under the home cage were collected, dried for a day, segregated, and weighed to obtain the correct amounts of kaolin and food intakes. The number of wheel turns was recorded after the running episode. In the following graphs and text, the average scores will be reported as arithmetic means with standard errors ( $S E \mathrm{~s}$ ) across subjects. A one-way repeated measures analysis of variance (ANOVA) or a paired $t$ test was applied to each data set having a day factor. Although statistical decisions were based on an alpha error level of $p<.05$, marginal effects $(.05 \leq p<.10)$ are also mentioned so as not to miss any possible trends of the independent variables. For simplicity, the standardized effect size will be reported only when the $p$ was less than .10.

\section{Ethical considerations}

All treatments of this and following experiments were approved by the Animal Care and Use Committee of Kwansei Gakuin University, based on a Japanese law and the guideline published by the Science Council of Japan.

\section{Results and discussion}

Running in activity wheels (45 min per day) gradually generated pica in rats up to the sixth day. The carryover effect was similar in amount whether the rats were exposed to the locked wheels or in the associatively neutral cages. The details are reported below.

\section{Kaolin intake}

As in previous studies conducted in my laboratory (Nakajima, 2016a, 2016b; Nakajima \& Katayama, 2014) and elsewhere (e.g., Rudd, Yamamoto, Yamatodani, \& Takeda, 2002), the rats consumed some kaolin in the beginning of the experiment, reflecting exploratory sampling of novel objects (Barnett, 1956). However, it gradually decreased to the nearzero level, as depicted in the left section in Fig. 1: An ANOVA applied to the baseline data yielded a marginal day effect, $F(4$, $28)=2.54, p=.062, \eta_{\mathrm{p}}{ }^{2}=.266$. The center section in Fig. 1 shows that wheel running gradually generated pica behavior 


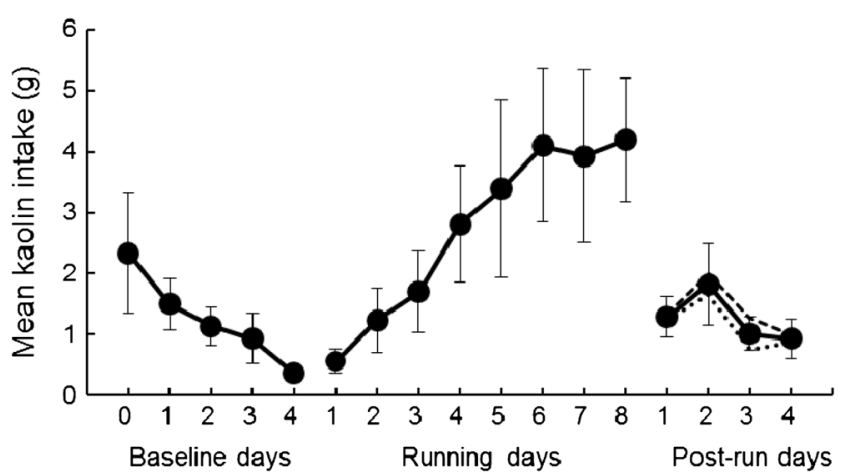

Fig. 1 Mean $( \pm S E)$ amount of kaolin intake across the successive days of Experiment 1. The kaolin pellets were available for $23 \mathrm{hr}$ in the home cages. In order to illustrate the effect of daily treatment in the experimental room on kaolin intake, the data are shown as a function of the days of treatment rather than the days of measurement. The first data plot is on Day 0, because the kaolin pellets were available from the day before the beginning of the baseline treatment. The broken and dotted lines on the postrunning days respectively represent the two subgroups exposed to the locked wheels and the empty cages. Their error bars were omitted for simplicity

up to the sixth day and leveled off thereafter: An ANOVA yielded a significant effect of day, $F(7,49)=4.59, p<.001$, $\eta_{\mathrm{p}}^{2}=.396$

The kaolin intake dropped immediately after the running opportunity was lost, as shown in the right section of Fig. 1. However, the effect of running on kaolin intake was somewhat carried over after the running treatment. For example, the rats consumed significantly more kaolin on the first postrunning day than on the last baseline day, paired $t(7)=$ $2.38, p=.049, r=.669$. Notably, the amount of kaolin intake was not affected by whether the rats were kept for $45 \mathrm{~min}$ in the locked wheels or in the empty cages: the broken line of Fig. 1 indicates the average intakes of the rats kept in the locked wheels on the odd days and in the empty cages on the even days, while the dotted line shows the averages of the rats assessed in the reversed order. For statistical analysis, the data were summarized to a 2 (context: locked wheel vs. empty cage $) \times 2$ (2-day cycle) fashion. On the first cycle, rats kept in the locked wheel and those in the empty cages, respectively, consumed kaolin of $1.4 \pm 0.6$ and $1.1 \pm 0.3 \mathrm{~g}$; the corresponding values on the second cycle were $1.6 \pm 0.5$ and $0.9 \pm 0.3 \mathrm{~g}$. An ANOVA yielded no significant main or interactive effects, $F_{\mathrm{s}}(1,7)<1.60, p \mathrm{~s}>.247$. A supplementary $2 \times 2$ Bayesian ANOVA using the statistical software JASP (JASP Team, 2017) with a default uniform distribution of prior model probabilities revealed that the null hypothesis was about 3 times more likely than the claim that the kaolin clay intake was affected by the context, $\mathrm{BF}_{01}=2.91$. The Bayes factor of the null hypothesis against the cycle model was $\mathrm{BF}_{01}=1.15$.

The statistically equivalent kaolin intakes of the two context conditions imply that the Pavlovian conditioning played little or no role in the carryover effect observed here.
Furthermore, the large drop in kaolin intake observed in the rats kept in the locked wheels in testing strongly indicates that the pica had been generated not by wheel confinement itself but by running in them (see also Nakajima \& Katayama, 2014, for another piece of evidence supporting this claim).

\section{Food intake}

The intake of food pellets slightly decreased in the baseline phase, $F(4,28)=2.77, p=.047, \eta_{\mathrm{p}}{ }^{2}=.283$. The averages were $24.1 \pm 0.8,24.0 \pm 0.8,22.9 \pm 0.8,23.7 \pm 1.0$, and $22.6 \pm 1.0 \mathrm{~g}$, from Day 0 to the fourth baseline days. The same trend was observed in the running phase, $F(7,49)=7.66, p<.001, \eta_{\mathrm{p}}{ }^{2}=$ .523. The averages were $24.6 \pm 0.7,21.6 \pm 0.8,22.4 \pm 0.5$, $22.0 \pm 0.4,21.9 \pm 0.5,21.3 \pm 0.6,21.7 \pm 0.4$, and $21.0 \pm 0.6 \mathrm{~g}$, from the first to the eighth running days. The intake was statistically unchanged in the in the postrunning phase, $F<1$. The averages were $22.4 \pm 0.5,23.0 \pm 0.8,22.4 \pm 0.7$, and 23.0 $\pm 0.7 \mathrm{~g}$, from the first to the fourth postrunning days. The small decrease of food consumption during the running phase has been also reported by previous studies on running-based pica (Nakajima, 2016a; Nakajima \& Katayama, 2014), and it might have reflected activity-based anorexia (Lattanzio \& Eikelboom, 2003). This possibility, however, must be viewed with caution because of the same decreasing trend in the baseline phase.

\section{Wheel turn}

The number of wheel turns was statistically unchanged throughout the running phase, $F<1$. The averages were 191 $\pm 11,196 \pm 10,197 \pm 8,192 \pm 10,193 \pm 9,194 \pm 11,184 \pm 8$, and $180 \pm 11$ turns per session, from the first to the eighth running days.

\section{Experiment 2}

Experiment 1 revealed that the number of daily sessions to obtain the asymptotic pica based on 45-min running is six in the preparation of the current study. Although the exact number of daily sessions to attain the asymptotic taste avoidance based on 45-min running is unknown, it is reasonable to assume that six sessions are enough to obtain the sizable avoidance, by considering the published experiments administered with 30-min running in our laboratory (e.g., Hayashi, Nakajima, Urushihara, \& Imada, 2002; Nakajima, 2008, 2014, 2015a). Accordingly, Experiment 2 was designed to monitor pica and taste avoidance during the 6-day running phase. Because the taste avoidance was measured by recording consumption of flavored solution as in our previous studies, rats were water-deprived in this and following experiments. 


\section{Method}

\section{Subjects and apparatus}

Two sets of 16 experimentally naïve male rats (Slc: Wistar/ ST) were purchased from the same supplier as in Experiment 1. One rat in the first replication set was too small compared with the others (2.24 standard deviations lower than the mean body weight) and rapidly lost its weight by $12 \%$ during the baseline phase, and then excluded from the experiment, resulting the total number of subjects of Experiment 2 being reduced to 31 .

The concurrent demonstration of running-based pica and taste avoidance was expected to be, and in fact turned out to be, achieved with a typical sample size of eight rats, but a larger sample was employed in Experiment 2 to examine correlations between several measures of pica and taste avoidance. The minimum number of subjects to be employed for assessment of Pearson correlations is 26, according to G*Power 3 (Faul, Erdfelder, Lang, \& Buchner, 2007) with effect size $=.50$, alpha $=.05$, tail $=2$, and power $=.80$. As the number of available wheels was eight, 32 rats $(8$ rats $\times 2$ squads $\times 2$ replications) were prepared, but one of the rats was excluded from the experiment as noted above. With the sample of 31 rats, the critical value of correlation coefficient at $p=$ .05 significance level is .355 .

The animals were housed with food and kaolin pellets as in Experiment 1, but water in the home cages was restricted to 1 hr per day as noted below. Tap water in the home cage was provided by the built-in nozzle noted in Experiment 1. After daily measurement of body weights, the rats were transferred to the experimental room of Experiment 1, which now had eight drinking cages (copies of the home cages) on a table. Tap water or sweet flavored water ( $0.2 \%$ sodium saccharin) was provided via a glass bottle with a metal spout inserted from the center of the cage ceiling. The end of the spout was $16.5 \mathrm{~cm}$ above the cage floor. When two bottles were used, they were separated $8 \mathrm{~cm}$ apart. The room also had the eight wheels as in Experiment 1.

\section{Procedure}

In each replication, all experimental procedures were administered with two squads of eight rats each on successive days. The first squad had a daily 15 -min fluid access starting at 1020 $\mathrm{hr}$, whereas the second squad had the same daily treatment at $1320 \mathrm{hr}$. The rats were also given a 1-hour tap water access in the home cages $1 \mathrm{hr}$ and $40 \mathrm{~min}$ after the start of the daily session. Since the replication and squad factors had no significant effect on any dependent measures, these factors were collapsed in the data analyses reported here.

All rats were deprived of water on Day 0. On the next 4 days (Days 1-4: baseline phase), they were adapted to drink tap water for $15 \mathrm{~min}$ from the bottles in the drinking cages. Thereafter, the rats remained there for another $45 \mathrm{~min}$ before being returned to the home cages. On the next 6 days (Days 510: conditioning phase), all rats were allowed to run freely in the unlocked wheels for $45 \mathrm{~min}$ immediately after a 15 -min access to sweet saccharin solution. The saccharin intakes was monitored as the primary measure of taste avoidance. As the secondary measure of taste avoidance, two-bottle choice testing was administered on the next 2 days (Days 11-12: test phase). One bottle contained the saccharin solution, whereas the other contained tap water. The left-right positions of the bottles were counterbalanced across rats and days in each squad. The rats were kept in the drinking cages for $45 \mathrm{~min}$ before being returned to the home cages.

After returning to the baseline for 3 days (Days 13-15), the final treatment was administered. In order to confirm that the rats were competent to show nausea-based pica, they were ipinjected on a day (Day 16) with $0.15 \mathrm{M} \mathrm{LiCl}$ at $2 \%$ of body weight immediately after a 15 -min access to tap water. Notably, we have reported that this dose is effective in generating pica (Nakajima \& Katayama, 2014).

\section{Measurement and analysis}

In addition to the amounts of food and kaolin consumed in the home cages, the intake of fluid in the experimental room was measured by weighing each bottle before and after the drinking period with an electric balance to the nearest $0.1 \mathrm{~g}$. The number of wheel turns was recorded as in Experiment 1. Statistical analyses were the same as in Experiment 1.

\section{Results and discussion}

Pica and taste avoidance were concurrently generated by wheel running. Furthermore, a weakly positive, though statistically marginal, relationship was observed between the pica and taste avoidance. The details are reported below.

\section{Kaolin intake}

Figure 2 illustrates the daily changes in kaolin consumption along the time course of this experiment. The error bars are shorter than those in Experiment 1 (Fig. 1) because of the larger sample size of this experiment. Parenthetically, the amounts of individual difference variance were similar to those in Experiment 1. For example, the standard deviation of kaolin intake on the sixth running day was 3.3 in this experiment, while it was 3.4 in Experiment 1.

As observed in Experiment 1, the rats showed some pica behavior in the beginning of the first baseline phase (the first section of Fig. 2), reflecting exploratory sampling of novel objects, and it gradually decreased to the near-zero level: An ANOVA applied to the baseline data yielded a significant day 


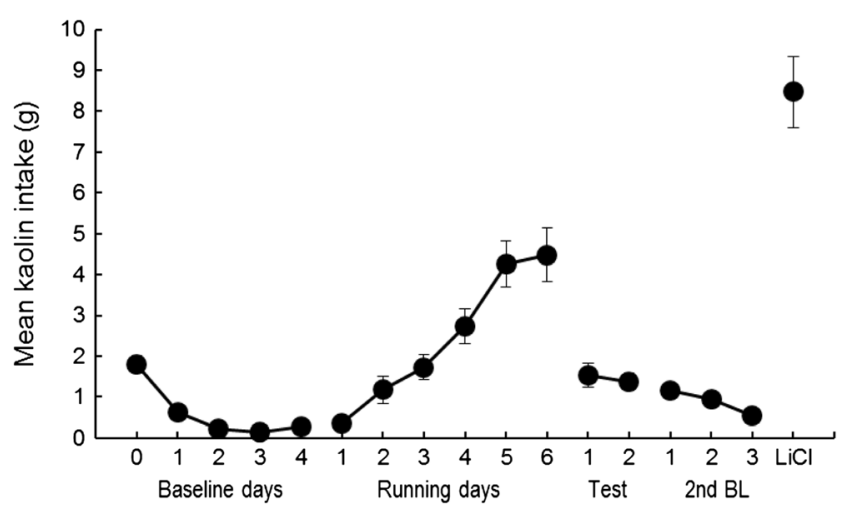

Fig. 2 Mean $( \pm S E)$ amount of kaolin intake across the successive days of Experiment 2. See the caption of Fig. 1 for details. Some error bars are smaller than the symbols

effect, $F(4,120)=39.79, p<.001, \eta_{\mathrm{p}}^{2}=.570$. The second section in Fig. 2 shows that wheel running gradually generated pica behavior up to the last day, $F(5,150)=36.86, p<.001$, $\eta_{\mathrm{p}}{ }^{2}=.551$. It is notable that this is the first demonstration of running-based pica with water-deprived rats, because tap water was freely available to all animals in Experiment 1 and in the previous studies (Nakajima, 2016a; Nakajima \& Katayama, 2014).

The kaolin intake dropped during the choice test phase, as shown in the third section of Fig. 2, with some carryover effect. Averaged over the 2 test days, the rats consumed 1.5 $\pm 0.2 \mathrm{~g}$ kaolin, and this score was significantly less than the intake of the last running day, paired $t(30)=6.35, p<.001, r=$ .758 , but significantly higher than the intake of the last baseline day, paired $t(30)=5.43, p<.001, r=.705$. In the second baseline phase, the mean kaolin intake slightly decreased over the 3 days (the fourth section of Fig. 2), $F(2,60)=8.19, p<$ $.001, \eta_{\mathrm{p}}{ }^{2}=.214$. The kaolin consumption was greatly increased by $\mathrm{LiCl}$ injection, as shown in the rightmost data point of Fig. 2: Tt was not only higher than the intake of the last days of the second baseline phase, paired $t(30)=9.50, p<$ $.001, r=.867$, but well above the intake of the last running day, paired $t(30)=6.69, p<.001, r=.774$.

\section{Food intakes}

The average food intakes were $13.3 \pm 0.3,15.6 \pm 0.5,17.0 \pm$ $0.3,17.4 \pm 0.4$, and $17.6 \pm 0.3 \mathrm{~g}$, from Day 0 to the fourth baseline days, $F(4,120)=35.36, p<.001, \eta_{\mathrm{p}}{ }^{2}=.541$. The relatively small intakes having the increasing trend, compared with the large intakes having the slightly decreasing trend observed in Experiment 1, were probably because rats were under water deprivation in this experiment but not in Experiment 1. The same, though weak, increasing trend was observed in the running phase: The averages were $17.6 \pm 0.3$, $17.8 \pm 0.4,17.3 \pm 0.3,17.3 \pm 0.3,18.1 \pm 0.3$, and $18.3 \pm 0.3 \mathrm{~g}$, from the first to the sixth running days, $F(5,120)=10.47, p<$ $.001, \eta_{\mathrm{p}}^{2}=.304$.

The food intakes on the two test days $(18.1 \pm 0.3$ and $18.3 \pm$ $0.3 \mathrm{~g}$ ) were statistically equivalent, paired $t(30)=1.17, p=$ .251. The food consumption was also statistically unchanged over the second baseline days $(18.6 \pm 0.3,19.0 \pm 0.3$, and 18.8 $\pm 0.3 \mathrm{~g}), F(2,60)=2.23, p=.116$. The rats consumed a significantly small amount of food after the $\mathrm{LiCl}$ injection $(16.6 \pm 0.3 \mathrm{~g})$, compared with the intake on the preceding baseline day, paired $t(30)=6.12, p<.001, r=.746$. This was expected owing to the $\mathrm{LiCl}$-induced illness.

\section{Fluid intake}

Tap water intakes during the baseline days were $6.5 \pm 0.3,8.8$ $\pm 0.4,9.1 \pm 0.3$, and $8.9 \pm 0.3 \mathrm{~g}$, from the first to the fourth baseline days: An ANOVA yielded a significant main effect of day, $F(3,90)=30.06, p<.001, \eta_{\mathrm{p}}{ }^{2}=.500$, suggesting the adaptation of drinking in rats. As shown in Fig. 3, the rats learned to avoid drinking the saccharin due to running in the wheels: An ANOVA yielded a significant day effect, $F(5,150)$ $=19.33, p<.001, \eta_{\mathrm{p}}{ }^{2}=.392$. It is remarkable that consumption of the same solution would gradually increase over days if the bottle access were not followed by running (cf. Hayashi et al., 2002; Masaki \& Nakajima, 2010; Nakajima, 2014; Nakajima, Kumazawa, Ieki, \& Hashimoto, 2012).

Figure 4 summarizes the mean saccharin and tap water intakes averaged over the 2 test days, because the day factor had no effect on the test data. The rats clearly avoided saccharin, paired $t(30)=8.10, p<.001, r=.829$. Notably, rats would prefer $0.2 \%$ saccharin solution to tap water if they had not received conditioning treatment (Hayashi et al., 2002; Masaki \& Nakajima, 2004a, 2005, 2006, 2010; Nakajima, 2014, 2015b). Tap water intakes during the second baseline days and the $\mathrm{LiCl}$ day were $11.2 \pm 0.4,11.2 \pm 0.4,11.5 \pm 0.4$, and $10.8 \pm 0.6 \mathrm{~g}$, respectively, from the first to the fourth baseline days: An ANOVA yielded no significant day effect, $F<1$.

\section{Wheel turn}

The number of wheel turns gradually increased during the running days. The averages were $126 \pm 10,148 \pm 11,150 \pm$ $9,153 \pm 10,146 \pm 10$, and $156 \pm 11$ turns per daily session, from the first to the sixth running days: An ANOVA yielded a significant day effect, $F(5,150)=3.54, p=.005, \eta_{\mathrm{p}}{ }^{2}=.106$.

\section{Correlations between running, pica, and taste avoidance}

The upper-right section of Table 1 summarizes Pearson correlation coefficients $(r)$ of major index of wheel running, pica, 


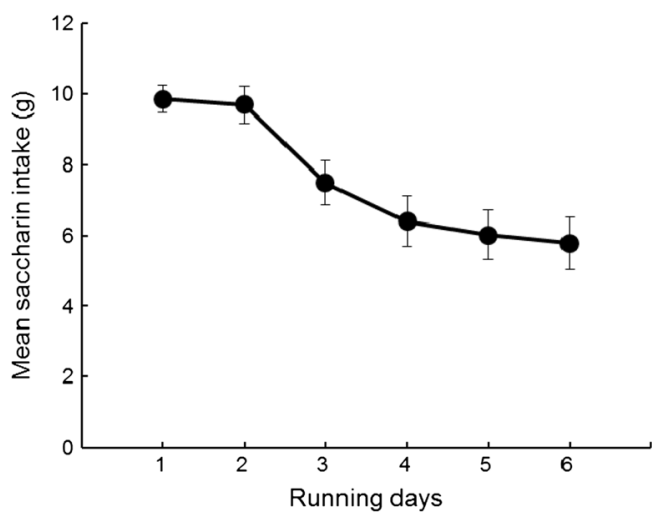

Fig. 3 Mean $( \pm S E)$ amount of saccharin solution intake in the conditioning phase of Experiment 2. The saccharin solution was available in the experimental room for $15 \mathrm{~min}$, which is followed by a 45-min wheel running

and taste avoidance, with the Bayes factors of the null hypothesis against the individual correlations $\left(\mathrm{BF}_{01}\right)$. The amounts of running and running-based pica are, respectively, represented by the number of wheel turns and the kaolin intake per running day (Nakajima, 2016a). The LiCl-based pica is shown as the kaolin intake after the $\mathrm{LiCl}$ injection. The primary index of running-based taste avoidance is the cumulative saccharin intake on the second day and onward, ${ }^{2}$ because the sequential saccharin-running pairing of a given day should affect the saccharin intakes of the following days. The secondary index of taste avoidance was from the choice test by calculating the saccharin preference ratio, which is the ratio of saccharin solution intake to total fluid intake. The lower the ratio value, the stronger is the avoidance estimated. A single ratio was directly calculated from the 2-day fluid intakes of each rat for statistical analyses. ${ }^{3}$ The average saccharin preference ratio calculated in this way was $0.22 \pm 0.03$. The kaolin intake on Day 0 (i.e., exploratory sampling) and the saccharin intake on the first running day (i.e., unconditioned saccharin preference) are also included in Table 1.

The first entry row of Table 1 shows that the amount of running had little effect on the kaolin and saccharin intakes. It is noteworthy that Nakajima (2016a) has reported that the magnitude of pica is a positive function of the amount of running if the latter is experimentally manipulated by changing the length of unlocked wheel confinement. It is also the case for running-based taste avoidance (Hayashi et al., 2002;

\footnotetext{
${ }^{2}$ Other indices of running-based taste avoidance, such as the saccharin intake on the last running day, the percentage decrease of saccharin intake in the running phase, and the suppression ratio proposed by Archer and Sjödén (1979) were also calculated, but they are not shown here because they were highly correlated with the cumulative saccharin intake adopted in Table 1 and thus redundant.

${ }^{3}$ The saccharin preference was unchanged across the test days: The average ratios separately calculated for the 2 test days were both $0.22 \pm 0.04$.
}

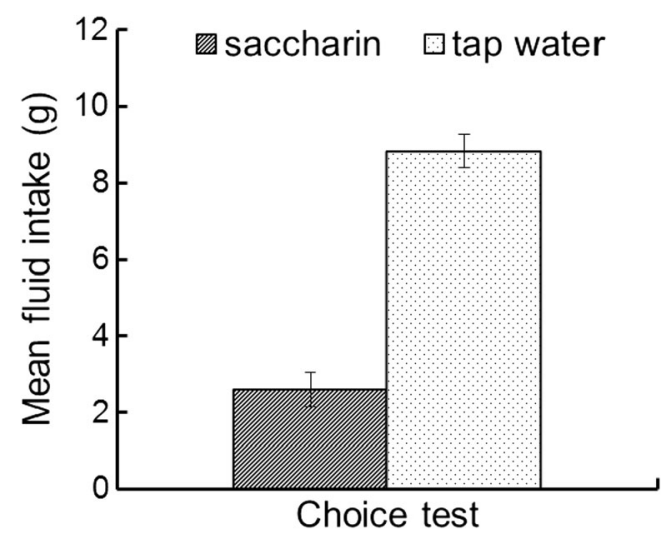

Fig. 4 Mean $( \pm S E)$ intakes of saccharin solution and tap water averaged over 2 test days of Experiment 2

Masaki \& Nakajima, 2006). The present results, however, are consistent with the finding that the magnitude of taste avoidance is not easily predicted by the amount of running if the latter is not manipulated experimentally (Nakajima, 2014). Most likely, individual differences in sensitivity to effect of running are so large as to mask an intrinsic linear runningnausea relationship.

The kaolin and saccharin intakes during the running days were negatively correlated $(r=-.346)$, suggesting a positive relationship between running-based pica and taste avoidance as expected, although it just missed the significance level, ${ }^{4} p=.056, \mathrm{BF}_{01}=0.79$. The other index of taste avoidance, the saccharin preference ratio, also negatively correlated with the kaolin intake $(r=-.234)$, but this correlation was far from the significance, $p=$ $.204, \mathrm{BF}_{01}=2.07$. Notably, the two indices of runningbased taste avoidance were weakly correlated $(r=.378, p$ $\left.=.036, \mathrm{BF}_{01}=0.55\right)$.

The most striking correlation was found between the running-based and LiCl-based kaolin intakes $(r=.731, p$ $\left.<.001, \mathrm{BF}_{01}=1.35 \times 10^{-4}\right)$. Notably, the correlation remained statistically significant even if Bonferroni correction for multiple testing was applied. One cannot attribute it to the individual differences in unconditional, general proneness to show pica behavior, because the kaolin intake on Day 0 was not correlated with these measures. Hence, the most likely account is that the common underlying physiological state (probably nausea) was induced by running and $\mathrm{LiCl}$ injection, and it invoked pica behavior.

\footnotetext{
${ }^{4}$ By considering the exploratory nature of this experiment, the error rate per comparison, rather than the family-wise error rate, is reported to minimize Type II error.
} 
Table 1. Correlations between indices of wheel running, pica, and taste avoidance

\begin{tabular}{|c|c|c|c|c|c|c|c|c|}
\hline & & $\begin{array}{l}\text { Wheel } \\
\text { All run days }\end{array}$ & $\begin{array}{l}\text { Kaolin } \\
\text { BL Day } 0\end{array}$ & $\begin{array}{l}\text { Kaolin } \\
\text { All run days }\end{array}$ & $\begin{array}{l}\text { Kaolin } \\
\text { LiCl-based }\end{array}$ & $\begin{array}{l}\text { Saccharin } \\
1 \text { st day }\end{array}$ & $\begin{array}{l}\text { Saccharin } \\
\text { 2nd onward }\end{array}$ & $\begin{array}{l}\text { Saccharin } \\
\text { Choice }\end{array}$ \\
\hline Wheel & $\begin{array}{l}\text { All run days } \\
\left(\mathrm{BF}_{01}\right)\end{array}$ & - & $\begin{array}{l}-.236 \\
(2.34)\end{array}$ & $\begin{array}{l}-.041 \\
(4.38)\end{array}$ & $\begin{array}{l}-.183 \\
(2.81)\end{array}$ & $\begin{array}{l}-.079 \\
(4.11)\end{array}$ & $\begin{array}{l}-.234 \\
(2.09)\end{array}$ & $\begin{array}{l}-.172 \\
(2.98)\end{array}$ \\
\hline Kaolin & $\begin{array}{l}\text { BL Day } 0 \\
\left(\mathrm{BF}_{01}\right.\end{array}$ & $\begin{array}{l}-.089 \\
(4.32)\end{array}$ & - & $\begin{array}{l}-.005 \\
(4.48)\end{array}$ & $\begin{array}{l}-.048 \\
(4.34)\end{array}$ & $\begin{array}{l}-.040 \\
(4.38)\end{array}$ & $\begin{array}{l}.164 \\
(3.08)\end{array}$ & $\begin{array}{l}.004 \\
(4.48)\end{array}$ \\
\hline Kaolin & $\begin{array}{l}\text { All run days } \\
\left(\mathrm{BF}_{01}\right)\end{array}$ & $\begin{array}{l}.066 \\
(4.59)\end{array}$ & $\begin{array}{l}.143 \\
(3.47)\end{array}$ & - & $\begin{array}{l}.731 \\
\left(1.35^{*} 10^{-4}\right)\end{array}$ & $\begin{array}{l}.006 \\
(4.48)\end{array}$ & $\begin{array}{l}-.346 \\
(0.79)\end{array}$ & $\begin{array}{l}-.234 \\
(2.07)\end{array}$ \\
\hline Kaolin & $\begin{array}{l}\text { LiCl-based } \\
\left(\mathrm{BF}_{01}\right)\end{array}$ & $\begin{array}{l}-.183 \\
(2.81)\end{array}$ & $\begin{array}{l}-.048 \\
(4.34)\end{array}$ & $\begin{array}{l}.731 \\
\left(1.35 * 10^{-4}\right)\end{array}$ & - & $\begin{array}{l}.187 \\
(2.76)\end{array}$ & $\begin{array}{l}-.061 \\
(4.26)\end{array}$ & $\begin{array}{l}-.277 \\
(1.50)\end{array}$ \\
\hline Saccharin & $\begin{array}{l}\text { 1st run day } \\
\left(\mathrm{BF}_{01}\right)\end{array}$ & $\begin{array}{l}-.004 \\
(4.95)\end{array}$ & $\begin{array}{l}.024 \\
(4.90)\end{array}$ & $\begin{array}{l}.060 \\
(4.66)\end{array}$ & $\begin{array}{l}.187 \\
(2.76)\end{array}$ & - & $\begin{array}{l}.264 \\
(1.67)\end{array}$ & $\begin{array}{l}-.040 \\
(4.38)\end{array}$ \\
\hline Saccharin & $\begin{array}{l}\text { 2nd onward } \\
\left(\mathrm{BF}_{01}\right)\end{array}$ & $\begin{array}{l}-.160 \\
(3.18)\end{array}$ & $\begin{array}{l}.127 \\
(3.75)\end{array}$ & $\begin{array}{l}-.304 \\
(0.94)\end{array}$ & $\begin{array}{l}-.061 \\
(4.24)\end{array}$ & $\begin{array}{l}.316 \\
(0.82)\end{array}$ & - & $\begin{array}{l}.378 \\
(0.55)\end{array}$ \\
\hline Saccharin & $\begin{array}{l}\text { Choice } \\
\left(\mathrm{BF}_{01}\right)\end{array}$ & $\begin{array}{l}-.106 \\
(4.08)\end{array}$ & $\begin{array}{l}-.037 \\
(4.84)\end{array}$ & $\begin{array}{l}-.243 \\
(1.74)\end{array}$ & $\begin{array}{l}-.277 \\
(1.50)\end{array}$ & $\begin{array}{l}-.047 \\
(4.77)\end{array}$ & $\begin{array}{l}.401 \\
(0.25)\end{array}$ & - \\
\hline
\end{tabular}

Notes. The upper-right section presents correlation coefficients calculated using data of Experiment $2(n=31)$. Inclusion of the data of the kaolin group of Experiment 3 results in similar scores as displayed in the lower-left section $(n=38$ in total). Because LiCl-based kaolin intakes were not measured in Experiment 3, the scores concerning this index were identical to those in Experiment 2 as shown in italics

\section{Experiment 3}

De Jonghe et al. (2009) claimed that pica behavior alleviates visceral discomfort induced by cisplatin. If this had been the case for the discomfort induced by running in Experiment 2, the increased kaolin intake would have weakened the potential of running to further establish taste avoidance. This might be the reason why the correlation between pica and taste avoidance was not so strong in Experiment 2. Experiment 3 tested this possibility by measuring running-based taste avoidance in rats that could consume kaolin and rats without kaolin. If kaolin has some alleviating (or remedial) effect on runninginduced discomfort, then the former rats should show weaker taste avoidance than the latter rats.

After the baseline phase of no running, two groups of rats were allowed to drink saccharin solution immediately followed by running voluntarily in activity wheels for 6 days. One of the groups was given kaolin in the home cages throughout the experiment, whereas the other group was not. As in Experiment 2, sweet saccharin solution was presented immediately before running, and thus its intake was monitored as a measure of taste avoidance. In addition, a posttraining choice test between saccharin and water was administered as another measure of taste avoidance. Experiment 3 was mainly concerned with whether the two groups of rats differ in the degree of taste avoidance. The weak avoidance in rats kept with kaolin compared with the no-kaolin rats would imply that kaolin has a remedial effect on running-induced discomfort in rats.

\section{Method}

\section{Subjects and apparatus}

A new set of 16 experimentally naïve male rats (Slc: Wistar/ ST) were housed and maintained as in Experiment 2, although the containers filled with kaolin pellets were installed only for half of the animals. The setting of the experimental room was the same as in Experiment 2.

\section{Procedure, measurement, and analysis}

All procedures in the experimental room were conducted on successive days with two squads of eight rats each. The first squad had a daily 15-min fluid access starting at $1020 \mathrm{hr}$, whereas the second squad had the same daily treatment at $1320 \mathrm{hr}$. The rats were given a 1-hour tap water access in the home cages $1 \mathrm{hr}$ and $40 \mathrm{~min}$ after the start of the daily session. Each squad consisted of four rats from two treatment groups (i.e., kaolin and no-kaolin groups). Since the squad factor had no significant effect on any dependent measures, this factor was collapsed in the data analyses reported below. The experimental procedure was identical to that of Experiment 2, except that the LiCl-treatment was not administered in this experiment. The measurement and analyses were the same as in Experiment 2, but a split-plot factorial ANOVAs or an independent $t$ tests was applied to the data when analyzing the group factor. 


\section{Results and discussion}

Wheel running generated pica behavior in rats kept with kaolin pellets. It also caused saccharin avoidance to the same extent as in rats kept without kaolin pellets. In other words, kaolin had no remedial effect on running-induced discomfort in rats. The details are reported below.

\section{Kaolin intake}

As in the preceding experiments, the rats kept with kaolin pellets engaged in pica in the beginning of the experiment, but it gradually decreased to the near-zero level (the left section of Fig. 5): An ANOVA applied to the baseline data yielded a significant day effect, $F(4,24)=3.61, p=.019, \eta_{\mathrm{p}}{ }^{2}$ $=.375$. The right section in Fig. 5 shows that wheel running gradually generated pica behavior, $F(5,30)=4.11, p=.006$, $\eta_{\mathrm{p}}^{2}=.406$

\section{Food intake}

The availability of kaolin in the home cage did not affect the intake of food pellets. A 2 (group) $\times 5$ (day) ANOVA, applied to the baseline data, yielded a significant main effect of day, $F(4,52)=17.47, p<.001, \eta_{\mathrm{p}}{ }^{2}=.573$, whereas the group factor had no significant main or interactive effect on food intake, $F \mathrm{~S}<1$. The averages collapsed over the group factor were $14.9 \pm 0.5,16.3 \pm 0.5,17.7 \pm 0.3,18.1 \pm 0.4$, and $17.8 \pm$ $0.3 \mathrm{~g}$, from Day 0 to the fourth days. Food consumption slightly changed during the running phase. A 2 (group) $\times 6$ (day) ANOVA yielded a significant main effect of day, $F(5,65)=$ $10.67, p<.001, \eta_{\mathrm{p}}{ }^{2}=.451$. Neither the main effect of group, $F$ $<1$, nor the interaction, $F(5,65)=1.04, p=.402$, was significant. The averages collapsed over the group factor were 19.4 $\pm 0.3,18.1 \pm 0.3,18.5 \pm 0.2,18.3 \pm 0.3,17.9 \pm 0.3$, and $18.1 \pm$ $0.4 \mathrm{~g}$, from the first to the sixth running days.

\section{Fluid intake}

The two groups did not differ in tap water intake during the baseline days: A 2 (group) $\times 4$ (day) ANOVA yielded a significant main effect of day, $F(3,39)=3.15, p=.036, \eta_{\mathrm{p}}{ }^{2}=$ .195 , suggesting the adaptation of drinking in rats, but neither the main effect of group, $F(1,13)=2.58, p=.132$, nor the interaction, $F(3,39)=1.16, p=.339$, was significant. The averages collapsed over the group factor were $7.6 \pm 0.6,9.8$ $\pm 0.7,8.1 \pm 0.4$, and $8.4 \pm 0.8 \mathrm{~g}$, from the first to the fourth baseline days.

The two groups were also similar during the conditioning days, as shown in Fig. 6. A 2 (group) $\times 5$ (day) ANOVA yielded a significant main effect of day, $F(5,65)=14.96, p$ $<.001, \eta_{\mathrm{p}}{ }^{2}=.535$, demonstrating acquisition of conditioned taste avoidance, whereas the group factor had no significant

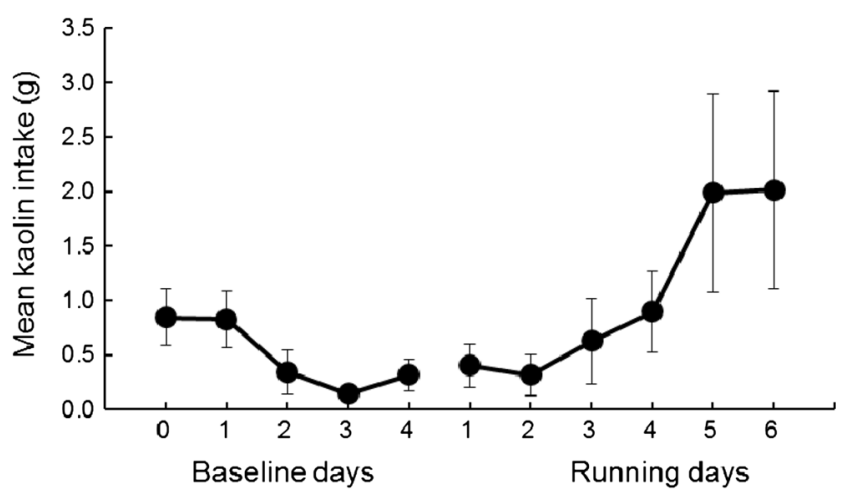

Fig. 5 Mean $( \pm S E)$ amount of kaolin intake across the successive days of Experiment 3. See the caption of Fig. 1 for details

main or interactive effect on saccharin intake, $F \mathrm{~S}<1$. To underpin the claim of null group difference, a supplementary $2 \times 6$ Bayesian ANOVA was performed. The Bayes factor of the null hypothesis against the main research hypothesis (i.e., the group factor) was $\mathrm{BF}_{01}=2.34$, hence representing at least anecdotal evidence for the null hypothesis against the claim that the availability of kaolin affects taste avoidance learning. Parenthetically, the Bayes factor of the null hypothesis against the day model was $\mathrm{BF}_{01}=2.01 \times 10^{-9}$, reflecting the clear acquisition of taste voidance across days.

Figure 7 summarizes the group mean saccharin and tap water intakes averaged over the 2 test days, because the day factor had no effect. The rats clearly avoided saccharin whether they had been kept with or without kaolin: a 2 (group) $\times 2$ (fluid: saccharin vs. water) ANOVA yielded a significant main effect of fluid, $F(1,13)=38.20, p<.001, \eta_{\mathrm{p}}{ }^{2}=.746$, implying saccharin avoidance, but the main effect of group was far from significance, $F<1$. The Group $\times$ Fluid interaction was also nonsignificant, $F(1,13)=3.17, p=.098, \eta_{\mathrm{p}}{ }^{2}=.196$. The group effect on the choice data was also examined by the saccharin preference ratio. As in Experiment 2, a single ratio was directly calculated from the 2-day fluid intakes of each rat

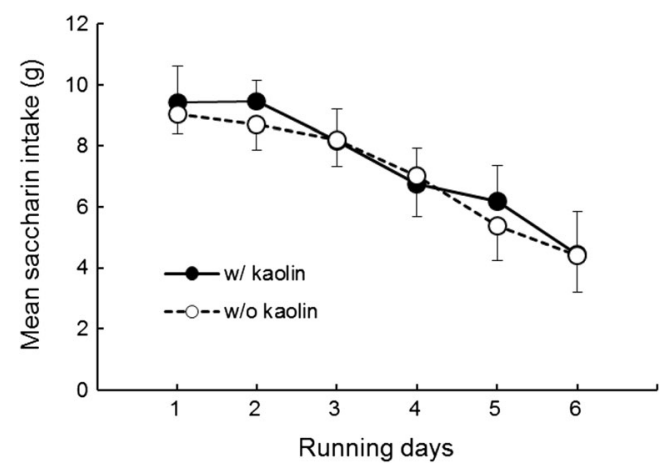

Fig. 6 Mean amount of saccharin solution intake in the conditioning phase of Experiment 3, separately shown for the rats kept with kaolin and the rats without kaolin in the home cages. Bars of SEs are shown on either side for clarity. See the caption of Fig. 2 for other details 


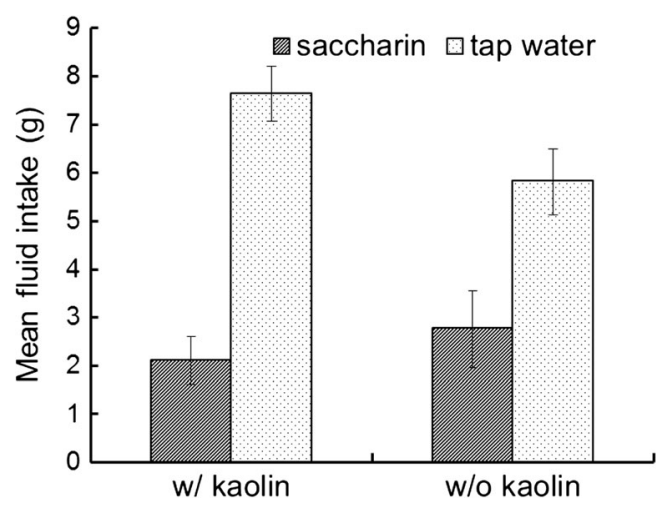

Fig. 7 Mean $( \pm S E)$ intakes of saccharin solution and tap water averaged over 2 test days of Experiment 3. The 2-day average data are shown separately for the rats kept with kaolin and the rats without kaolin in the home cages

for statistical analyses. The average ratios of the kaolin and no-kaolin groups, respectively, were $0.21 \pm 0.04$ and $0.30 \pm$ 0.07 , which did not statistically differ from each other, $t(13)=$ $1.14, p=.275$. If anything, the pattern is opposite to what was expected from the hypothesis that kaolin reduces the severity of nausea. These results suggest that kaolin was not a remedy for running-induced discomfort.

\section{Wheel turn}

The two groups did not differ in the amount of running. A 2 (group) $\times 6$ (day) ANOVA yielded no significant main effects of group and day, and the interaction was also nonsignificant. The averages collapsed over the group factor were $101 \pm 12$, $112 \pm 12,123 \pm 16,116 \pm 13,131 \pm 14$, and $122 \pm 15$ turns per daily session, from the first to the sixth running days.

\section{Correlations between running, pica, and taste avoidance}

The experimental treatments for the rats in the kaolin group were identical to those for the subjects of Experiment 2 up to the choice preference test. Thus, the data of the kaolin group were added to the data set of Experiment 2 to examine the correlations between the behavioral measures with a much larger sample $(n=38)$. The results are summarized in the lower-left section of Table 1. As shown in the first entry column, the amount of running once again had little effect on the other measures. The kaolin and saccharin intake during the running days was negatively correlated ( $r=-.304)$, suggesting a weakly positive relationship between running-based pica and taste avoidance, but it failed to reach the significance level, $p=.064, \mathrm{BF}_{01}=0.94$. Furthermore, the other index of taste avoidance, the saccharin preference ratio, was not significantly correlated with the kaolin intake $(r=-.243, p=.141$, $\left.\mathrm{BF}_{01}=1.74\right)$. Notably, the two indices of running-based taste avoidance were weakly correlated $\left(r=.401, p=.012, \mathrm{BF}_{01}=\right.$
$0.25)$. The positive correlation in the saccharin intake between the first and the remaining days of the running phase $(r=.316)$ was marginally significant, $p=.054, \mathrm{BF}_{01}=0.86$. A similar positive correlation between preconditioning and postconditioning saccharin intake was reported by Archer and Sjödén (1979).

\section{Experiment 4}

So far, we have assumed that taste avoidance is based on nausea in rats. However, nausea is not necessary to establish taste avoidance (Grant, 1987; Parker, 2003, 2014; Parker et al., 2009). In other words, demonstration of taste avoidance does not guarantee by itself that rats felt nausea in training. Further, pica behavior might also be generated by nonemetic treatments. If this is the case, the argument that wheel running induces nausea in rats would be invalid. Experiment 4 was designed to examine this issue by exploring the effect of visceral pain on pica behavior. As noted in the introduction of this article, repeated injections of hypertonic saline evoke visceral pain and cause avoidance of paired taste (e.g., Hargrave \& Bolles, 1971; Lin et al., 2013; Sakai \& Yamamoto, 1997). Hence, in Experiment 4, pica behavior in rats was monitored to assess the effect of such injections. If pica is a genuine marker of nausea, it should be negligible in injected rats.

\section{Method}

\section{Subjects and apparatus}

Experiment 4 started after a 37-day recess with free food and water. Eight rats with a history of kaolin intake in Experiment 3 were excluded from the study, for fear of affecting kaolin intake in Experiment 4. Thus, eight rats that had been kept without kaolin in Experiment 3 were used as the subjects of Experiment 4. The experimental setup was the same as in the preceding experiments, though the wheels were not employed in Experiment 4. The tastes employed were sour, bitter, and salty solutions to minimize any effect of Experiment 3 where sweet solution (saccharin) had been used. Notably, these four tastes are perceptually independent from each other (Chandrashekar, Hoon, Ryba, \& Zuker, 2006). One concern is the possibility that the weakly bitter taste of saccharin (Dess, 1994) would facilitate conditioned taste avoidance with the bitter denatonium solution, but this was not the case in this experiment as noted below.

\section{Procedure}

All procedures in the experimental room were conducted in a single squad of eight rats, and the rats had a daily 15-min fluid access in the drinking cages starting at $1020 \mathrm{hr}$. Thereafter, the 
rats were kept in the drinking cages without any fluid for $45 \mathrm{~min}$ before being returned to the home cages, where they were given a 1-hour tap water access starting at $1200 \mathrm{hr}$.

On Day 0, kaolin pellet containers were installed, and all rats were deprived of water. On the first 2 days of baseline training (Days 1-2), all rats were readapted to drink tap water for $15 \mathrm{~min}$ from the bottles in the drinking cages. On the next 2 baseline days (Days 3-4), tap water was replaced with either sour $(0.1 \%$ citric acid) or bitter $(0.4 \mathrm{ppm}$ denatonium benzoate) solution to make it familiar to the rats; this solution was to be used later as a control taste in the choice test. The concentrations of these solutions were determined by a pilot study with other rats to produce equivalent amounts of initial intake by rats. From the next day for 4 days (Days 5-8), all rats were given an ip injection of $1.0 \mathrm{M}$ hypertonic saline at $0.5 \%$ of body weight immediately after a $15-\mathrm{min}$ access to a target solution (bitter or sour). The dose and number of injections (i.e., the conditioning trials) were identical to those of Lin et al. (2013, Experiment 2). It is also noteworthy that the number of conditioning trial was limited to four because of ethical concerns. For half of the rats, the control and target tastes, respectively, were sour and bitter, whereas the control-target combination was reversed for the remaining rats.

Two-bottle choice testing was administered on the next 2 days (Days 9-10) as the secondary measure of taste avoidance. One bottle contained the sour solution, whereas the other contained the bitter solution. The left-right positions of the bottles were counterbalanced across rats and days in each control-target combination subgroup. The choice of two tastes (sour vs. bitter), rather than a choice between the conditioned target taste and tap water, was used in this experiment in order to control unconditioned taste avoidance regarding these tastes.

In order to confirm that the subjects of Experiment 4 were competent to show nausea-based pica, they were ip-injected on the next day (Day 11) with isotonic but emetic $0.15 \mathrm{M} \mathrm{LiCl}$ at $2 \%$ of body weight immediately after a 15 -min access to salty $(1 \% \mathrm{NaCl})$ solution. On the following 2 days (Days 12 13), the rats were given salty solution for 15 min without any injection to assess the acquisition of taste avoidance.

\section{Measurement and analysis}

The measurement and analysis of the data were administered as the previous experiments, but Wilcoxon signed rank test, instead of paired $t$ test, was applied to the data, when they had a skewed distribution.

\section{Results and discussion}

Injection of hypertonic saline caused avoidance of the paired taste without generating any pica in rats. However, the same rats showed strong pica by an emetic $\mathrm{LiCl}$ injection, which also yielded avoidance of the taste consumed before the injection. The details are described below. The following sections begin with the fluid consumption data, because they would legitimate the usage of hypertonic saline as an agent for yielding taste avoidance.

\section{Fluid intake}

Figure 8 illustrates the daily changes in fluid consumption throughout the experiment. The two subgroups did not differ during the baseline days when the bottles contained tap water or a control taste solution: a 2 (subgroup) $\times 4$ (day) ANOVA yielded no significant main or interactive effect, $F \mathrm{~s}<1$. The two subgroups, however, differed in the intake of the target solution from the first hypertonic day and onward. Since the access to the fluid preceded the injection, the subgroup disparity on the first hypertonic day might imply that a bittersour and sour-bitter shifts caused differential contrast effects. More importantly for our research question, the fluid intake gradually and reliably decreased over days, indicating clear acquisition of taste avoidance by hypertonic saline injection. A 2 (subgroup) $\times 4$ (day) ANOVA yielded significant main effects of subgroup, $F(1,6)=12.94, p=.011, \eta_{\mathrm{p}}{ }^{2}=.683$, and day, $F(3,18)=31.10, p<.001, \eta_{\mathrm{p}}{ }^{2}=.838$, but their interaction failed to reach the significance, $F(3,18)=2.49, p=.093, \eta_{\mathrm{p}}{ }^{2}=$ 293.

Figure 9 depicts the choice test data based on the 2-day averages of target and control tastes, separately shown for the subgroups. The day factor was collapsed, because it had no main or interactive effect. Conditioned taste avoidance is evident whether the target was bitter or sour taste. A 2 (subgroup) $\times 2$ (fluid: target vs. control) ANOVA yielded a significant main effect of fluid, $F(1,6)=14.14, p=.009, \eta_{\mathrm{p}}{ }^{2}=$ .702. The main effect of subgroup was statistically nonsignificant, $F<1$, as was the interaction, $F(1,6)=2.10, p=.197$.

An injection of emetic $\mathrm{LiCl}$ endowed rats with strong avoidance of the paired salty solution as shown in the right section in Fig. 8. A 2 (subgroup) $\times 3$ (day) ANOVA yielded a significant main effect of day, $F(2,12)=25.56, p<.001, \eta_{\mathrm{p}}{ }^{2}=$ .810 , whereas the main effect of subgroup and the interaction were far from significance, $F \mathrm{~s}<1$. Post hoc analyses with Ryan's procedure (Howell, 2006) statistically supported the visual impression of the average data: The intake of the first salty day was larger than the remaining 2 days, which did not differ from each other.

\section{Kaolin intake}

Figure 10 illustrates the daily changes in kaolin consumption along the time course of this experiment. The factor of subgroup regarding physical identities of the control and target tastes (sour and bitter) was collapsed because it had no significant main or interactive effect on kaolin intake in any analysis 


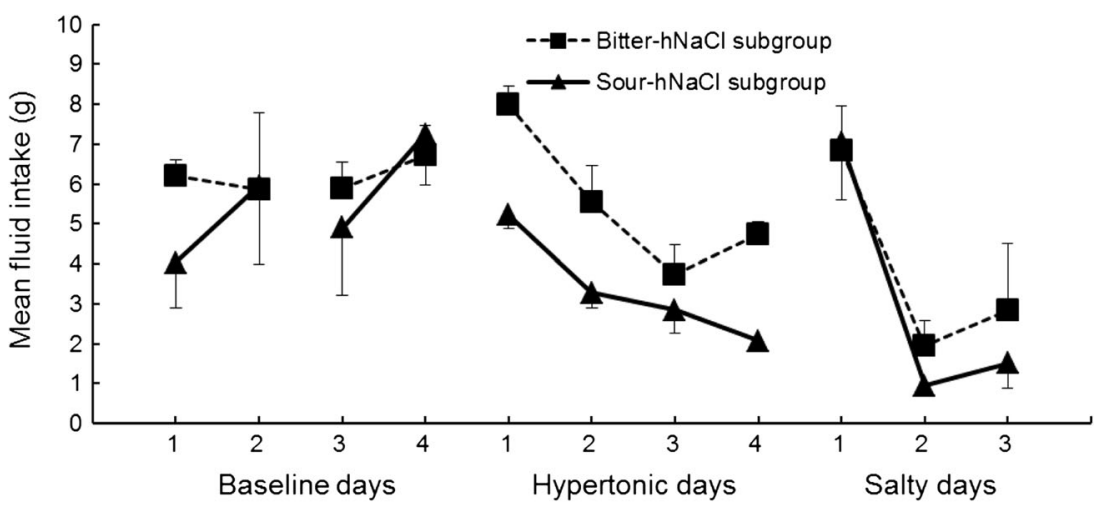

Fig. 8 Mean amount of fluid intake in Experiment 4. The data are separately shown for the rats receiving injections of $\mathrm{hNaCl}$ (hypertonic saline) after drinking either bitter or sour solution on hypertonic days. The fluid was tap water on the first and second baseline days for all rats, whereas it is sour or bitter solution (taste not used for the subsequent hypertonic days) on the third and fourth baseline days. The fluid was salty water on salty days for all rats. Bars of SEs are shown on either side for clarity. Some error bars are smaller than the symbols noted below. The rats showed some pica behavior on the first day of the first baseline phase as in the preceding experiments. Notably, the subjects of Experiment 4 were the rats kept without kaolin in Experiment 3. Thus, they first encountered the kaolin pellets in the beginning of Experiment 4. Kaolin intake ceased to the nearest zero level on the second baseline day and onward. A 2 (subgroup) $\times 5$ (day) ANOVA, applied to the baseline data, yielded a significant main effect of day, $F(4$, 24) $=12.32, p<.001, \eta_{\mathrm{p}}{ }^{2}=.673$. Neither the main effect of subgroup, $F(1,6)=2.82, p=.144$, nor the interaction, $F(4,24)$ $=1.61, p=.205$, was significant.

The middle section in Fig. 10 shows that injections of hypertonic saline failed to generate pica behavior. Kaolin intake was negligible throughout the phase. The kaolin intake averaged over the 4 hypertonic days was statistically equivalent to the intake averaged over the last 2 baseline days, paired $t(7)=$ $1.09, p=.313$. Although the kaolin intake averaged over the hypertonic days was slightly larger for the rats given the bitter solution than the remaining rats $(0.2 . \pm 0.1$ vs. $0.0 \pm 0.0 \mathrm{~g})$, the difference was minuscule and the main effect of subgroup in the 2 (subgroup) $\times 4$ (day) ANOVA failed to reach the significance level, $F(1,6)=5.73, p=.054, \eta_{\mathrm{p}}{ }^{2}=.488$. The main

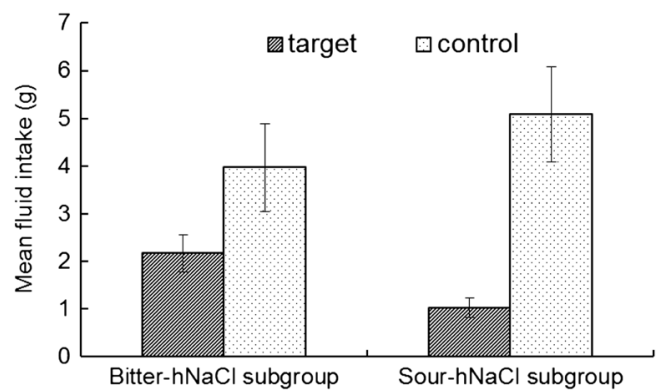

Fig. 9 Mean $( \pm S E)$ intakes of hNaCl-paired target solution and unpaired control solution in the choice test of Experiment 4. The 2-day average data are shown separately for two subgroups, which differed in the physical identities of paired and unpaired tastes effect of day, $F(3,18)=2.43, p=.099, \eta_{\mathrm{p}}^{2}=.288$, and the interaction, $F(3,18)=2.85, p=.066, \eta_{\mathrm{p}}{ }^{2}=.322$, also failed to reach the significance. The possibility that much longer training with hypertonic saline would generate kaolin clay ingestion cannot be ignored, but seems improbable in view of the fact that there were any signs of incremental change in kaolin clay intake up to the fourth trial.

Kaolin intake was maintained low during the choice tests, but pica was evoked by an emetic $\mathrm{LiCl}$ injection as shown in the last data point in Fig. 10. All rats consumed large amounts of kaolin, as compared with the intake averaged over the preceding 2 choice test days: two-tailed Wilcoxon signed rank test, $T=0, n=8, p=.012, r=.630$.

\section{Food intake}

The amount of food intake slightly increased over the baseline phase. A 2 (subgroup) $\times 5$ (day) ANOVA yielded a significant main effect of day, $F(4,24)=19.57, p<.001, \eta_{\mathrm{p}}^{2}=.765$. Neither the main effect of subgroup nor the interaction was significant, $F \mathrm{~s}<1$. The averages collapsed over the subgroup

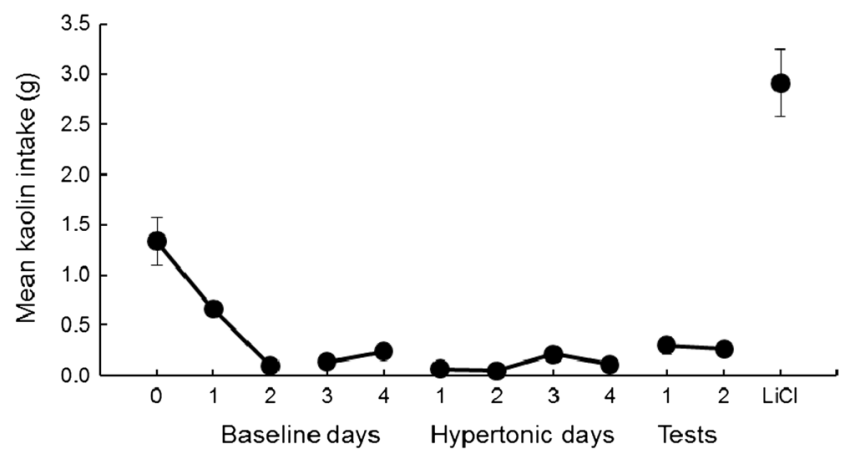

Fig. 10 Mean $( \pm S E)$ amount of kaolin intake across the successive days of Experiment 4. See the caption of Fig. 1 for details. Some error bars are smaller than the symbols 
factor were $12.5 \pm 1.0,14.7 \pm 1.0,15.8 \pm 0.3,15.8 \pm 0.3$, and $16.4 \pm 0.6 \mathrm{~g}$, from Day 0 to fourth days. Injections of hypertonic saline did not affect food consumption. A 2 (subgroup) $\times$ 4 (day) ANOVA yielded no significant main effects of subgroup, $F<1$, or day, $F(3,18)=1.65, p=.214$. The interaction was also nonsignificant, $F(3,18)=1.32, p=.299$. The averages of all rats were $16.3 \pm 0.3,16.0 \pm 0.6,14.8 \pm 0.6$, and 16.8 $\pm 0.6 \mathrm{~g}$, from the first to the fourth hypertonic days. In addition, food intake averaged over the 4 hypertonic days was statistically equivalent to the intake averaged over the last 2 baseline days, paired $t<1$.

Food intake was unchanged by administering the choice tests or by an emetic $\mathrm{LiCl}$ injection. The food intake averaged over the 2 test days $(15.9 \pm 0.5 \mathrm{~g})$ was statistically equivalent to the intake during the 23 -hour period after $\mathrm{LiCl}$ injection $(16.3 \pm 0.4 \mathrm{~g})$, paired $t<1$.

\section{General discussion}

As reported in the previous articles (Nakajima, 2016a; Nakajima \& Katayama, 2014), running in an activity wheel generated pica behavior in rats (Experiments 1, 2, and 3). Since the amount of pica (kaolin clay intake) was drastically dropped when the wheel was locked (Experiment 1), one must conclude that running, rather than wheel confinement itself, played the central role in the pica during the running phase. Notably, Nakajima and Katayama (2014) have also shown that wheel confinement did not generate pica behavior in rats.

Although we observed some pica in the home cages after they were confined into the locked wheels on postrunning days, the amount was similar to that observed after they were kept in the empty cages (Experiment 1). Thus, these are the carryover effects from the running phase rather than Pavlovian conditioned pica evoked by the contextual cues. The author is not prepared to offer any certain explanation for this kind of behavioral inertia or "resistance to change." A related question to be clarified is acquisition of pica over running days because this might be another case of "resistance to change." Otherwise, the strength of discomfort grows by repeated opportunities of wheel running. It is noteworthy that we have once claimed that the opponent-process theory of motivation (Solomon, 1980; Solomon \& Corbit, 1974) is applicable to rats' running-based place avoidance (Masaki \& Nakajima, 2008), where we speculated that repeated experience of running might evoke a initially large but gradually dwindling unpleasant physiological state as the "a-process" and a secondary but gradually developing pleasant state as the "b-process." The acquisition of pica across days, however, might demand inversion of the hedonic polarities of the opponent processes induced by running. In this framework, the acquisition of pica reflects development of the unpleasant nausea state across days. Although further research is required to test the validity of this new speculation, it is notable that a similar account has been proposed for mood changes with repeated use of addictive drugs (Koob \& Le Moal, 2001).

Wheel running concurrently generated pica and taste avoidance in Experiment 2, implying that the pica and taste avoidance were evoked by a common or similar physiological state (probably nausea). Although the correlation coefficient between the kaolin intake and the major index of taste avoidance was not high $(r=.346)$ and remained at a marginally significant level, this might be regarded as sufficiently strong by considering the fact that a similar low value was obtained even when we calculated the correlation between the two indices of taste avoidance $(r=.378)$. More surprising is a strong correlation between the amounts of running-based and LiCl-based pica ( $r=$ .731 ), suggesting the commonality between the physiological states induced by wheel the running and $\mathrm{LiCl}$ injection.

Experiment 3 has implicated that kaolin intake does not alleviate visceral discomfort induced by voluntary wheel running, because the magnitude of taste avoidance was equivalent between the rats to which kaolin was available and those to which it was unavailable. Probably, running-induced discomfort is fortuitously similar to toxin-induced nausea, and thus misguidedly provokes instinctive behavior of kaolin consumption in vain (Nakajima, 2016a; Nakajima \& Katayama, 2014). In other words, running-based pica is likely a product of body "fallacy" (or misperception). This claim, however, needs further investigation. For example, kaolin might possibly alleviate running-induced visceral discomfort in some fashion, but the physiology of running-based pica has not yet been elucidated.

Pica behavior seems to be a valid marker of nausea because it is easily invoked by an injection of emetic $\mathrm{LiCl}$ but not by injections of nonemetic hypertonic saline (Experiment 4). One cannot ascribe the inability of hypertonic saline to generate pica to its relative weakness as an aversive stimulus, because wheel running, which is much weaker than the hypertonic saline in producing taste avoidance, has reliably generated pica in Experiments 2 and 3 as well as in the previous studies (Nakajima, 2016a; Nakajima \& Katayama, 2014). Hence, Experiment 4 has segregated the two kinds of visceral discomforts (i.e., pain and nausea). In other words, visceral pain induced by hypertonic saline qualitatively differs from nausea induced by $\mathrm{LiCl}$, although hypertonic saline was effective in causing taste avoidance as demonstrated here and by other researchers (e.g., Andrews \& Braveman, 1975; Hargrave \& Bolles, 1971; Lin et al., 2013; Sakai \& Yamamoto, 1997). 
The hypothesis that visceral pain and nausea are different in physiology to cause taste avoidance accords with the report that aversive orofacial responses are generated by emetic $\mathrm{LiCl}$ injection but not by visceral pain induced by oral ingestion of lactose (Pelchat, Grill, Rozin, \& Jacobs, 1983). However, the finding of Lin et al. (2013) that injection of hypertonic saline reduces taste palatability measured by licking patterns in rats is contradictory to this hypothesis. Since the licking-pattern microstructure in rats has been considered a valid measure of gastrointestinal malaise in rats (e.g., Arthurs, Lin, Amodeo, \& Reilly, 2012; Baird et al., 2005; Dwyer, 2009; Lin et al., 2012), Lin et al. (2013) have concluded that hypertonic saline induces not only pain but also gastrointestinal malaise in rats (see also Lin et al., 2014, 2017). This conclusion appears to be incongruent with the result of Experiment 4 that hypertonic saline failed to generate pica, our marker of nausea in rats. Although further study is needed to resolve this discrepancy, a possible solution is to argue that nausea is more specific illness than broader symptoms of gastrointestinal malaise.

Another puzzle related to the present study is that swimming in water generates pica in rats (Nakajima, 2016b), implying that swimming induces visceral discomfort similar to nausea. Although swimming also endows rats with taste avoidance (Masaki \& Nakajima, 2004a, 2004b, 2005, 2010; Nakajima, 2004; Nakajima \& Masaki, 2004), failures of crosshabituation between swimming and running suggest that these two activities are qualitatively different in physiology to endow taste avoidance (Nakajima, 2015a). Therefore, the validity of pica as a behavioral marker of nausea should be further investigated before more definite statements can be made. It is possible that running and swimming differ in their sites of action. For instance, one may assume that running and swimming, respectively, induce malaise in upper and lower gastrointestinal tracts, and both of which yield pica. This hypothesis is currently under investigation in my laboratory.

We now know that pica is a useful behavioral marker of running-induced discomfort in rats. Future research will be directed to a finer-grained time-course analysis of daily kaolin intake for tracking the running-induced discomfort by using an automatic monitoring system, such as that developed by Yamamoto, Asano, Matsukawa, Imaizumi, and Yamatodani (2011). We also have learned that pica has little effect on running-based taste avoidance, but is this similarly the case for emetic-based taste avoidance? Mitchell et al. (1977) have reported a concurrent demonstration of pica and taste avoidance based on emetic cyclophosphamide injection, but they did not examine whether the pica alleviated the taste avoidance in rats. Because kaolin clay absorbs toxin (Dominy et al., 2004), prevents diarrhea (Beck et al., 1977), modifies nutrient absorption (Reichardt et al., 2009), and helps recovery from chemotherapy-induced anorexia (De Jonghe et al., 2009), pica behavior would alleviate malaise and then attenuate taste avoidance based on emetic drugs. This issue must be addressed in future work. In conclusion, the present study has brought us a step forward in understanding runningbased pica and taste avoidance, but it also confronts us with new challenges.

Acknowledgements This study was supported by JSPS KAKENHI Grant (15K04201) and MEXT Strategic Project to Support the Formation of Research Bases at Private Universities.

\section{References}

Andrews, P. L., \& Horn, C. C. (2006). Signals for nausea and emesis: Implications for models of upper gastrointestinal diseases. Autonomic Neuroscience, 125, 100-115. doi:https://doi.org/10. 1016/j.autneu.2006.01.008

Andrews, E. A., \& Braveman, N. S. (1975). The combined effects of dosage level and interstimulus interval on the formation of onetrial poison-based aversions in rats. Animal Learning \& Behavior, 3, 287-289. doi:https://doi.org/10.3758/BF03213446

Archer, T., \& Sjödén, P. O. (1979). Positive correlation between pre- and postconditioning saccharin intake in taste-aversion learning. Animal Learning \& Behavior, 7, 144-148. doi:https://doi.org/10.3758/ BF03209263

Arthurs, J., Lin, J. Y., Amodeo, L. R., \& Reilly, S. (2012). Reduced palatability in drug-induced taste aversion: II. Aversive and rewarding unconditioned stimuli. Behavioral Neuroscience, 126, 433-444. doi:https://doi.org/10.1037/a0027676

Aung, H., Mehendale, S., Chang, W. T., Wang, C. Z., Xie, J. T., \& Yuan, C. S. (2005). Scutellaria baicalensis decreases ritonavir-induced nausea. AIDS Research and Therapy, 2, 12. doi:https://doi.org/10. 1186/1742-6405-2-12

Aung, H. H., Mehendale, S. R., Xie, J. T., Moss, J., \& Yuan, C. S. (2004). Methylnaltrexone prevents morphine-induced kaolin intake in the rat. Life Sciences, 74, 2685-2691. doi:https://doi.org/10.1016/j.lfs. 2003.08.047

Baird, J. P., St John, S. J., \& Nguyen, E. A. N. (2005). Temporal and qualitative dynamics of conditioned taste aversion processing: Combined generalization testing and licking microstructure analysis. Behavioral Neuroscience, 119, 983-1003. doi:https://doi.org/10. 1037/0735-7044.119.4.983

Barnett, S. A. (1956). Behaviour components in the feeding of wild and laboratory rats. Behaviour, 9, 24-43. doi:https://doi.org/10.1163/ $156853956 \times 00237$

Beck, I. T., Jenkins, N., Thurber, L., \& Ambrus, J. L. (1977). Methods for the study of antidiarrheal agents: Study of commonly used protective and adsorbent agents. Journal of Medicine, 8, 135-158.

Boakes, R. A., \& Nakajima, S. (2009). Conditioned taste aversions based on running or swimming. In S. Reilly \& T. R. Schachtman (Eds.), Conditioned taste aversion: Behavioral and neural processes (pp. 159-78). New York, NY: Oxford University Press.

Chandrashekar, J., Hoon, M. A., Ryba, N. J., \& Zuker, C. S. (2006). The receptors and cells for mammalian taste. Nature, 444, 288-294. doi: https://doi.org/10.1038/nature05401

De Jonghe, B. C., Lawler, M. P., Horn, C. C., \& Tordoff, M. G. (2009). Pica as an adaptive response: Kaolin consumption helps rats recover from chemotherapy-induced illness. Physiology \& Behavior, 97, 87-90. doi: https://doi.org/10.1016/j.physbeh.2009.02.009.

Dess, N. K. (1994). Saccharin's aversive taste in rats: Evidence and implications. Neuroscience \& Biobehavioral Reviews, 17, 359-372. doi:https://doi.org/10.1016/S0149-7634(05)80113-7

Dominy, N. J., Davoust, E., \& Minekus, M. (2004). Adaptive function of soil consumption: An in vitro study modeling the human stomach 
and small intestine. Journal of Experimental Biology, 207, 319-324. doi:https://doi.org/10.1242/jeb.00758

Dwyer, D. M. (2009). Microstructural analysis of ingestive behaviour reveals no contribution of palatability to the incomplete extinction of a conditioned taste aversion. Quarterly Journal of Experimental Psychology, 62, 9-17. doi:https://doi.org/10.1080/ 17470210802215152

Dwyer, D. M. (2012). Licking and liking: The assessment of hedonic responses in rodents. Quarterly Journal of Experimental Psychology, 65, 371-394. doi:https://doi.org/10.1080/17470218. 2011.652969

Dwyer, D. M., Boakes, R. A., \& Hayward, A. J. (2008). Reduced palatability in lithium- and activity-based, but not in amphetamine-based, taste aversion learning. Behavioral Neuroscience, 122, 1051-1060. doi: https://doi.org/10.1037/a0012703

Eccles, S., Kim, E. M., \& O'Hare, E. (2005). Granisetron attenuates exercise-induced conditioned taste aversion in the rat. Appetite, 44, 325-328. doi:https://doi.org/10.1016/j.appet.2005.02.001

Faul, F., Erdfelder, E., Lang, A. G., \& Buchner, A. (2007). G*Power 3: A flexible statistical power analysis program for the social, behavioral, and biomedical sciences. Behavior Research Methods, 39, 175-191.

Garcia, J., Kovner, R., \& Green, K. F. (1970). Cue properties vs palatability of flavors in avoidance learning. Psychonomic Science, 20, 313-314. doi:https://doi.org/10.3758/BF03329085

Grant, V. L. (1987). Do conditioned taste aversions result from activation of emetic mechanisms?. Psychopharmacology, 93, 405-415. doi: https://doi.org/10.1007/BF00207227

Grant, V. L., McDonald, S. V., Sheppard, R. C., Caldwell, C. L., Heeley, T. H., Brown, A. R., \& Martin, G. M. (2012). Dissociation of conditioned taste avoidance from conditioned disgust reactions induced by wheel running in rats. Behavioural Processes, 90, 223-228. doi: https://doi.org/10.1016/j.beproc.2012.01.011

Grill, H. J., \& Norgren, R. (1978). The taste reactivity test: I. Mimetic responses to gustatory stimuli in neurologically normal rats. Brain Research, 143, 263-279. doi:https://doi.org/10.1016/00068993(78)90568-1

Hargrave, G. E., \& Bolles, R. C. (1971). Rat's aversion to flavors following induced illness. Psychonomic Science, 23, 91-92. doi:https:// doi.org/10.3758/BF03336022

Hayashi, H., Nakajima, S., Urushihara, K., \& Imada, H. (2002). Taste avoidance caused by spontaneous wheel running: Effects of duration and delay of wheel confinement. Learning and Motivation, 33, 390409. doi:https://doi.org/10.1016/S0023-9690(02)00004-8

Heth, C. D., Inglis, P., Russell, J. C., \& Pierce, W. D. (2001). Conditioned taste aversion induced by wheel running is not due to novelty of the wheel. Physiology \& Behavior, 74, 53-56. doi:https://doi.org/10. 1016/S0031-9384(01)00553-4

Horn, C. C., Kimball, B. A., Wang, H., Kaus, J., Dienel, S., Nagy, A., . . \& Andrews, P. L. (2013). Why can't rodents vomit? A comparative behavioral, anatomical, and physiological study. PLOS ONE, 8(4), e60537. doi:https://doi.org/10.1371/journal.pone.0060537

Howell, D. C. (2006). Statistical methods for psychology (6th ed.). Belmont, CA: Wadsworth.

JASP Team (2017). JASP (Version 0.8.1.2)[Computer software]. Amsterdam, Netherlands: JASP.

Koob, G. F., \& Le Moal, M. (2001). Drug addiction, dysregulation of reward, and allostasis. Neuropsychopharmacology, 24, 97-129. doi: https://doi.org/10.1016/S0893-133X(00)00195-0

Krane, R. V., \& Wagner, A. R. (1975). Taste aversion learning with a delayed shock US: Implications for the generality of the laws of learning. Journal of Comparative and Physiological Psychology, 88, 882-889. doi:https://doi.org/10.1037/h0076417

Lattanzio, S. B., \& Eikelboom, R. (2003). Wheel access duration in rats: I. Effects on feeding and running. Behavioral Neuroscience, 117, 496-504. doi:https://doi.org/10.1037/0735-7044.117.3.496
Lett, B. T., \& Grant, V. L. (1996). Wheel running induces conditioned taste aversion in rats trained while hungry and thirsty. Physiology \& Behavior, 59, 699-702. doi:https://doi.org/10.1016/0031-9384(95) 02139-6

Lin, J. Y., Arthurs, J., Amodeo, L. R., \& Reilly, S. (2012). Reduced palatability in drug-induced taste aversion: I. Variations in the initial value of the conditioned stimulus. Behavioral Neuroscience, 126 , 423-432. doi:https://doi.org/10.1037/a0027674

Lin, J. Y., Arthurs, J., \& Reilly, S. (2013). Reduced palatability in paininduced conditioned taste aversions. Physiology \& Behavior, 119, 79-85. doi:https://doi.org/10.1016/j.physbeh.2013.06.012

Lin, J. Y., Arthurs, J., \& Reilly, S. (2014). Conditioned taste aversion, drugs of abuse and palatability. Neuroscience \& Biobehavioral Reviews, 45, 28-45. doi:https://doi.org/10.1016/j.neubiorev.2014. 05.001

Lin, J. Y., Arthurs, J., \& Reilly, S. (2017). Conditioned taste aversions: From poisons to pain to drugs of abuse. Psychonomic Bulletin \& Review, 24, 335-351. doi:https://doi.org/10.3758/s13423-0161092-8

Masaki, T., \& Nakajima, S. (2004a). Swimming-induced taste aversion and its prevention by a prior history of swimming. Learning and Motivation, 35, 406-418. doi:https://doi.org/10.1016/j.lmot.2004. 04.003

Masaki, T., \& Nakajima, S. (2004b). Taste aversion learning induced by delayed swimming activity. Behavioural Processes, 67, 357-362. doi:https://doi.org/10.1016/j.beproc.2004.06.005

Masaki, T., \& Nakajima, S. (2005). Further evidence for conditioned taste aversion induced by forced swimming. Physiology \& Behavior, 84, 9-15. doi:https://doi.org/10.1016/j.physbeh.2004.09.022

Masaki, T., \& Nakajima, S. (2006). Taste aversion in rats induced by forced swimming, voluntary running, forced running, and lithium chloride injection treatments. Physiology \& Behavior, 88, 411-416. doi:https://doi.org/10.1016/j.physbeh.2006.04.013

Masaki, T., \& Nakajima, S. (2008). Forward conditioning with wheel running causes place aversion in rats. Behavioural Processes, 79, 43-47. doi:https://doi.org/10.1016/j.beproc.2008.04.006

Masaki, T., \& Nakajima, S. (2010). The effect of swimming experience on acquisition and retention of swimming-based taste aversion learning in rats. Learning and Motivation, 41, 32-47. doi:https:// doi.org/10.1016/j.lmot.2009.09.001

Mitchell, D., Laycock, J. D., \& Stephens, W. F. (1977a). Motion sicknessinduced pica in the rat. American Journal of Clinical Nutrition, 30, $147-150$.

Mitchell, D., Wells, C., Hoch, N., Lind, K., Woods, S. C., \& Mitchell, L. K. (1976). Poison induced pica in rats. Physiology \& Behavior, 17, 691-697. doi:https://doi.org/10.1016/0031-9384(76)90171-2

Mitchell, D., Winter, W., \& Morisaki, C. M. (1977b). Conditioned taste aversions accompanied by geophagia: Evidence for the occurrence of psychological factors in the etiology of pica. Psychosomatic Medicine, 39, 402-412.

Nakajima, S. (2004). Conditioned ethanol aversion in rats induced by voluntary wheel running, forced swimming, and electric shock: An implication for aversion therapy of alcoholism. Integrative Physiological \& Behavioral Science, 39, 95-104. doi:https://doi. org/10.1007/BF02734275

Nakajima, S. (2008). Effect of extra running on running-based taste aversion in rats. Behavioural Processes, 78, 470-472. doi:https://doi. org/10.1016/j.beproc.2008.01.018

Nakajima, S. (2014). Running-based taste aversion learning in five strains of rats. Physiology \& Behavior, 123, 200-213. doi: https://doi.org/ 10.1016/j.physbeh.2013.10.011

Nakajima, S. (2015a). Prior running, but not swimming, hinders runningbased taste aversion learning in rats. Learning and Motivation, 51, 1-10. doi:https://doi.org/10.1016/j.lmot.2015.02.003

Nakajima, S. (2015b). Swimming in flavored water leads to avoidance of that flavor in laboratory rats (Rattus norvegicus). International 
Journal of Comparative Psychology, 28. Retrieved from http:// escholarship.org/uc/item $/ 4 \mathrm{pm} 9 \mathrm{z} 01 \mathrm{k}$

Nakajima, S. (2016a). Running induces nausea in rats: Kaolin intake generated by voluntary and forced wheel running. Appetite, 106, 85-94. doi:https://doi.org/10.1016/j.appet.2016.05.009

Nakajima, S. (2016b). Swimming-based pica in rats. Behavioural Processes, 130, 1-3. doi:https://doi.org/10.1016/j.beproc.2016.06. 015

Nakajima, S., Hayashi, H., \& Kato, T. (2000). Taste aversion induced by confinement in a running wheel. Behavioural Processes, 49, 35-42. doi:https://doi.org/10.1016/S0376-6357(00)00071-1

Nakajima, S., \& Katayama, T. (2014). Running-based pica in rats. Evidence for the gastrointestinal discomfort hypothesis of runningbased taste aversion. Appetite, 83, 178-184. doi:https://doi.org/10. 1016/j.appet.2014.08.031

Nakajima, S., Kumazawa, G., Ieki, H., \& Hashimoto, A. (2012). Does conspecific fighting yield conditioned taste aversion in rats? The Psychological Record, 62, 83-90. doi:https://doi.org/10.1007/ BF03395788

Nakajima, S., \& Masaki, T. (2004). Taste aversion learning induced by forced swimming in rats. Physiology \& Behavior, 80, 623-628. doi: https://doi.org/10.1016/j.physbeh.2003.11.006

Nakajima, S., Urata, T., \& Ogawa, Y. (2006). Familiarization and crossfamiliarization of wheel running and $\mathrm{LiCl}$ in conditioned taste aversion. Physiology \& Behavior, 88, 1-11. doi:https://doi.org/10.1016/ j.physbeh.2006.02.006

Ossenkopp, K.-P., Parker, L. A., Limebeer, C. L., Burton, P., CrossMellor, S. K., \& Fudge, M. A. (2003). Vestibular lesions selectively abolish rotation-induced, but not lithium-induced, conditioned taste aversions (oral rejection responses) in rats. Behavioral Neuroscience, 117, 105-112. doi:https://doi.org/10.1037/07357044.117.1.105

Parker, L. A. (2003). Taste avoidance and taste aversion: Evidence for two different processes. Animal Learning \& Behavior, 31, 165-172. doi:https://doi.org/10.3758/BF03195979

Parker, L. A. (2014). Conditioned taste aversion learning. In F. K. McSweeney \& E. S. Murphy (Eds.), The Wiley Blackwell handbook of operant and classical conditioning (pp. 97-116). Oxford, UK: Wiley. doi:https://doi.org/10.1002/9781118468135.ch5

Parker, L. A., Limebeer, C. L., \& Rana, S. A. (2009). Conditioned disgust, but not conditioned taste avoidance, may reflect conditioned nausea in rats. In S. Reilly \& T. R. Schachtman (Eds.), Conditioned taste aversions: Behavioral and neural processes (pp. 92-113). New York, NY: Oxford University Press.

Pelchat, M. L., Grill, H. J., Rozin, P., \& Jacobs, J. (1983). Quality of acquired responses to tastes by Rattus norvegicus depends on type of associated discomfort. Journal of Comparative Psychology, 97, 140-153. doi:https://doi.org/10.1037/0735-7036.97.2.140

Reichardt, F., Habold, C., Chaumande, B., Ackermann, A., EhretSabatier, L., Le Maho, Y., . . . \& Lignot, J. H. (2009). Interactions between ingested kaolinite and the intestinal mucosa in rat: Proteomic and cellular evidences. Fundamental \& Clinical Pharmacology, 23, 69-79.

Rudd, J. A., Yamamoto, K., Yamatodani, A., \& Takeda, N. (2002). Differential action of ondansetron and dexamethasone to modify cisplatin-induced acute and delayed kaolin consumption ("pica") in rats. European Journal of Pharmacology, 454, 47-52. doi: https://doi.org/10.1016/S0014-2999(02)02472-X

Sakai, N., \& Yamamoto, T. (1997). Conditioned taste aversion and c-Fos expression in the rat brainstem after administration of various USs. NeuroReport, 8, 2215-2220. doi:https://doi.org/10.1097/00001756199707070-00025

Solomon, R. L. (1980). The opponent-process theory of acquired motivation: The costs of pleasure and the benefits of pain. American Psychologist, 35, 691-712. doi:https://doi.org/10.1037/0003-066X. 35.8.691

Solomon, R. L., \& Corbit, J. D. (1974). An opponent-process theory of motivation: I. Temporal dynamics of affect. Psychological Review, 81, 119-145. doi:https://doi.org/10.1037/h0036128

Takeda, N., Hasegawa, S., Morita, M., Horii, A., Uno, A., Yamatodani, A., \& Matsunaga, T. (1995). Neuropharmacological mechanisms of emesis: I. Effects of antiemetic drugs on motion-and apomorphineinduced pica in rats. Methods and Findings in Experimental and Clinical Pharmacology, 17, 589-590.

Takeda, N., Hasegawa, S., Morita, M., \& Matsunaga, T. (1993). Pica in rats is analogous to emesis: An animal model in emesis research. Pharmacology Biochemistry and Behavior, 45, 817-821. doi:https:// doi.org/10.1016/0091-3057(93)90126-E

Tohei, A., Kojima, S. I., Ikeda, M., Hokao, R., \& Shinoda, M. (2011). Effects of cyclophosphamide on the kaolin consumption (pica behavior) in five strains of adult male rats. Journal of Veterinary Medical Science, 73, 901-906. Doi:https://doi.org/10.1292/jvms. 10-0433

Yamamoto, K., Asano, K., Matsukawa, N., Imaizumi, M., \& Yamatodani, A. (2011). Time-course analysis of pica in rats using an automatic feeding monitoring system. Journal of Pharmacological and Toxicological Methods, 63, 30-34. doi:https://doi.org/10.1016/j. vascn.2010.04.011

Yamamoto, K., Takeda, N., \& Yamatodani, A. (2002). Establishment of an animal model for radiation-induced vomiting in rats using pica. Journal of Radiation Research, 43, 135-141. doi:https://doi.org/10. 1269/jrr.43.135 